\title{
Catheter-based treatment of tricuspid regurgitation: state of the art
}

\author{
Marcel Santaló-Corcoy ${ }^{1}$, Lluís Asmarats ${ }^{1}$, Chi-Hion Li ${ }^{2}$, Dabit Arzamendi ${ }^{1}$ \\ ${ }^{1}$ Division of Interventional Cardiology, ${ }^{2}$ Division of Cardiac Imaging, Hospital de la Santa Creu i Sant Pau, Universitat Autònoma de Barcelona, \\ Barcelona, Spain \\ Contributions: (I) Conception and design: None; (II) Administrative support: None; (III) Provision of study materials or patients: None; (IV) \\ Collection and assembly of data: None; (V) Data analysis and interpretation: None; (VI) Manuscript writing: All authors; (VII) Final approval of \\ manuscript: All authors. \\ Correspondence to: Dabit Arzamendi. Division of Interventional Cardiology, Hospital de la Santa Creu i Sant Pau, Sant Antoni M. Claret, 167, 08025, \\ Barcelona, Spain. Email: darzamendi@santpau.cat.
}

\begin{abstract}
Tricuspid regurgitation is a highly prevalent condition, with detrimental effects on long-term survival. However, it has been historically neglected, and only surgically addressed when symptomatic diuretic agents proved insufficient to alleviate congestion. Besides, mortality rates of isolated tricuspid regurgitation surgery have been persistently high, even in contemporary series. This has led to the advent of a myriad of transcatheter tricuspid valve interventions mimicking current surgical technologies, for which a comprehensive imaging work-up holds the key for proper patient selection and intraprocedural monitoring. Although initially designed for compassionate use patients, growing experience and encouraging results of these less-invasive technologies are broadening the spectrum of beneficiaries. In this review, we will focus on the current picture of transcatheter tricuspid valve interventions, with special interest on the current understanding of pathoanatomic tricuspid regurgitation progression, preprocedural multimodality imaging and the latest experience on the different transcatheter devices.
\end{abstract}

Keywords: Tricuspid regurgitation (TR); transcatheter therapies

Submitted Dec 15, 2019. Accepted for publication Jan 20, 2020.

doi: 10.21037/atm.2020.03.219

View this article at: http://dx.doi.org/10.21037/atm.2020.03.219

\section{Introduction}

Tricuspid regurgitation (TR) is a highly prevalent condition, with an estimate prevalence of 1.6 million people in the United States with moderate to severe TR (1). Its detrimental effect on long term survival is well established (2), with a recent meta-analysis showing moderate to severe TR is associated with an increased mortality risk, independent of pulmonary pressures and right ventricle (RV) dysfunction (3). Surgery has traditionally been the mainstay treatment for severe TR, with diuretics being the palliative alternative to alleviate systemic congestion. In fact, current guidelines recommend early tricuspid valve repair in patients with tricuspid annular dilatation undergoing left-sided cardiac surgery, even in mild TR, as well as in symptomatic isolated severe tricuspid regurgitation $(4,5)$. However, only a minority of patients undergo isolated tricuspid valve (TV) operations, ranging from $11 \%$ to $15 \%$ of all TV surgeries, as shown in two contemporary 10-year United States national registry $(6,7)$. This reluctance to perform isolated TV surgery might be explained by the persistently high in-hospital mortality after these procedures, ranging from $8.8 \%$ to $9.7 \%$ (Figure 1). These negative outcomes may be related to high comorbidity burden of the patients and late referral rather than the risk of isolated TV surgery itself (8). In sum, this scenario has led to the recent development of a myriad of transcatheter tricuspid valve interventions (TTVIs), which aim to prove their feasibility, safety and effectiveness in the treatment of TR, as well as a paradigm shift to a prompt referral of less ill patients with significant TR. We provide an updated review on the understanding of functional TR and its percutaneous treatment. 


\section{TV anatomy}

A deep knowledge of the anatomy and spatial relationships of TV apparatus is of paramount importance when planning transcatheter interventions (9) (Figure 2). The TV is the largest of all heart valves, and consists of three leaflets (septal, anterior

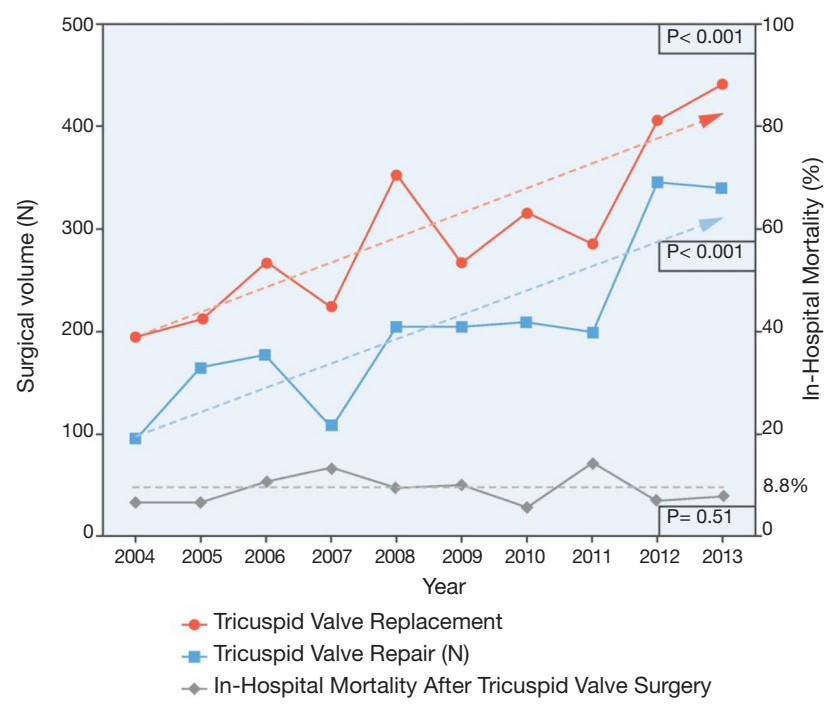

Figure 1 Annual trends in surgical volume and mortality for isolated tricuspid valve surgery. As the total amount of both isolated tricuspid valve replacement and repair has risen in from 2004 to 2013 , in-hospital mortality has held steady in a median $8.8 \%$ ratio. Reproduced with permission from (6). and posterior) separated by three commissures. Compared with the mitral valve, the leaflets are thinner and more radiolucent. They are supported by the papillary muscles, which are usually organized into three groups: anterior, posterior and septal, being the former the dominant one. It is implanted near the apex and fuses with the moderator band. There are four important anatomic structures surrounding the TV which risk interference during intervention: (I) the atrioventricular node (which sits in the apex of the triangle of Koch, demarcated by the septal leaflet, the tendon of Todaro and the coronary sinus); (II) the bundle of His, which runs underneath the membranous septum 3 to $5 \mathrm{~mm}$ from the anteroseptal commissure; (III) the right coronary artery [which can follow a non-favorable course of $<2 \mathrm{~mm}$ distance from the annulus at the level of the posterior leaflet in one out of four patients (10)] and (IV) the non-coronary sinus of Valsalva.

\section{Etiology and pathophysiology}

TR can be classified in primary (organic) and secondary (functional). The former is uncommon and can be found in patients with congenital heart disease (mainly Ebstein's anomaly), pacemaker or ICD lead-induced leaflet damage, trauma (endomyocardial biopsy, chest wall trauma) carcinoid syndrome, endomyocardial fibrosis or endocarditis.

Functional tricuspid regurgitation (FTR) accounts for $>90 \%$ of all TR, where the regurgitation is due to progressive annulus dilatation and flattening, and lack of

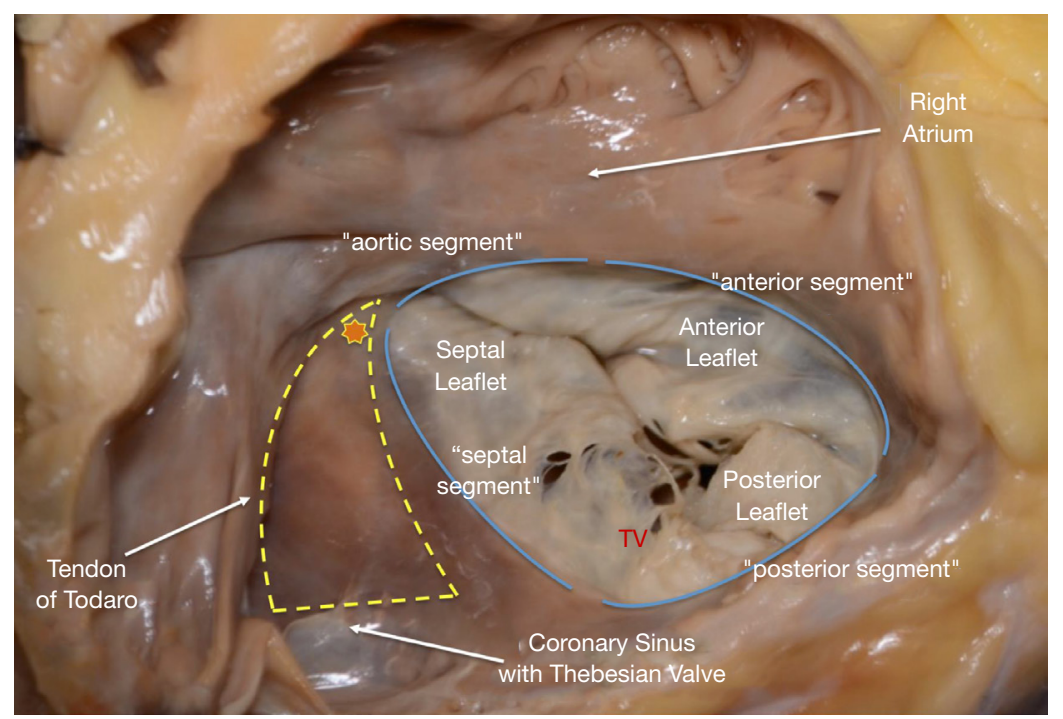

Figure 2 Anatomy of tricuspid valve and surrounding structures. Yellow triangle depicts the triangle of Koch, delineated by the septal leaflet, the tendon of Toaro and the coronary sinus. The star marks the location of the atrioventricular node. Reproduced with permission from (9). 


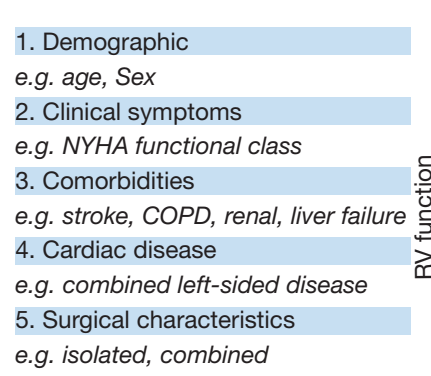

Patient risk stratification

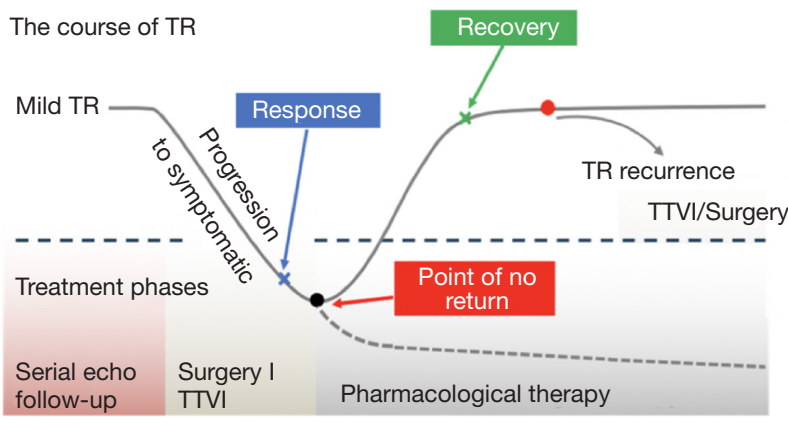

Pathological change of tricuspid valve/RV

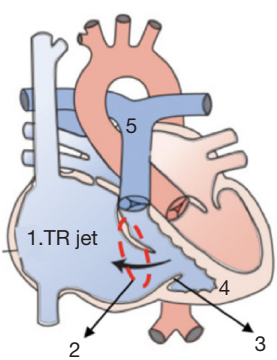

1. TR severity

2. Annulus size

3. Tricuspid valve morphology

4. $R V$ remodelling

5. Pulmonary vascular resistance

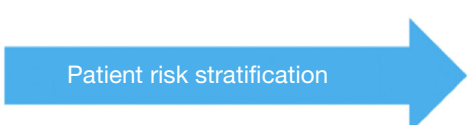

Heart team decision-making

Cardiac pathological remodelling

Figure 3 Stepwise heart team decision-making for treatment of tricuspid regurgitation. The implementation of a complete patient risk stratification in addition to a comprehensive imaging work-up for the assessment of cardiac pathological remodeling may aid in the accurate heart team to provide appropriate treatment (surgical, minimal invasive surgical, transcatheter, pharmacological, or palliative). Reproduced with permission from (14).

leaflet coaptation, rather than organic leaflet defect (11). Two distinct scenarios can be described: (I) dilatation of both the right ventricle and tricuspid annulus due to leftsided heart disease; pulmonary hypertension independent of left-sided pathology; or right ventricular infarctions with remodeling; and (II) atrial fibrillation leading to annulus dilatation (12). Whichever the cause, the development of TR often entails the onset of a vicious cycle where it begets more TR by means of right ventricular dilatation and dysfunction, tricuspid annular dilatation, tricuspid leaflet tethering and subsequent tricuspid regurgitation worsening. Of note, the tricuspid annulus and leaflets adapt differently in left-sided heart disease than in atrial fibrillation-induced TR. In the former, the annulus expands predominantly along the anterolateral border and there is a more significant tethering of the leaflets, whereas in the latter there is a larger, planar and circular expansion of the tricuspid annulus predominantly along the posterior border, with less significant tethering (13).

If left untreated, patients with long-lasting severe TR fail to respond to diuretic therapy and show signs and symptoms of intractable right heart failure, primarily being systemic congestion (edema, ascites, hepatosplenomegaly) and reduced cardiac output (fatigue, dizziness, early satiety) until end-organ dysfunction appears with impact on mortality.

\section{Imaging work-up}

\section{TV anatomy and RV function}

An initial two-dimensional (2D) transthoracic echocardiography (TTE) remains the cornerstone imaging modality to assess TV anatomy and function. Evaluation of the anatomic characteristics of the three leaflets can provide insight of possible causes of primary tricuspid regurgitation, while the measurement of a dilated tricuspid annulus with normal leaflets may lead to a functional TR diagnosis. Three-dimensional (3D) TTE plays an important role in assessing leaflets features, while specific 3D transesophageal echocardiography (TEE) provides unique information of the complex anatomic $3 \mathrm{D}$ changes in the tricuspid annulus geometry as FTR develops (Figure 3). Moreover, 3D TEE has the unique capability of obtaining a short-axis transgastric plane of the TV, simultaneous visualization of the three leaflets, commissures and the attachment of the tricuspid annulus. Moreover, as geometry of the tricuspid annulus changes with its dilation, 3D offers more reliable and accurate measurements (15). Finally, TEE is of paramount importance for intraprocedural monitoring of TTVIs.

Thanks to its spatial and temporal resolution, CT offers very valuable information when planning tricuspid intervention. In particular, the critical addition of CT scan 
is a reliable and precise measurements of certain variables needed for TTVI, such as tricuspid annulus and right ventricular geometry, landing zone geometry, subvalvular apparatus and surrounding structures to delineate possible impediments, and vascular access assessment $(16,17)$.

As discussed earlier, right ventricular dilatation has an intrinsic role in the development and worsening of TR. A comprehensive study of its size and function is crucial in its diagnosis and in planning of TR transcatheter repair. Due to its triangular shape in the coronal plane and crescent shape in the transversal plane, the assessment of RV size and function requires multiple views in 2D TTE. Current recommendation indicates that a basal $\mathrm{RV}$ diameter $>41 \mathrm{~mm}$ or a mid-cavity diameter $>35 \mathrm{~mm}$ indicate $\mathrm{RV}$ enlargement and a longitudinal linear dimension of $\mathrm{RV}$ $>83 \mathrm{~mm}$ indicates $\mathrm{RV}$ dilation. In terms of RV function, RV fractional area change $<35 \%$ and tricuspid annular plane systolic excursion (TAPSE) $<17 \mathrm{~mm}$ indicate RV dysfunction (18).

\section{TR severity}

Echocardiography remains the cornerstone imaging modality to assess the presence and severity of TR. Current North American and European Association of Echocardiography guidelines categorize TR in three stages (mild, moderate and severe) by means of qualitative, semiquantitative and quantitative methods (4,5) (Table 1). However, the advent of the new TTVIs have proven this grading to be incomplete. Indeed, in end-stage TR patient populations undergoing percutaneous repair, the effective regurgitant orifice area (EROA) may often double the upper-limit margin for severe TR. When treated with percutaneous techniques, the resultant EROA continued to fall well into "severe" criteria, despite significant improvements in symptoms and quality of life, as well as an increase in forward stroke volume (20). Thus, a novel expansion of the severe TR grading system has been proposed so as to adequately grade TR severity and better assess the efficacy of TTVI, with the inclusion of massive and torrential grades (21) (Table 2).

In sum, the role of imaging work-up can be summarized in providing accurate assessment of TR etiology and severity, TA dimensions and RV function and remodeling (leading to a proper patient selection) as well as reliable measurements of the specific variables needed for each TTVI. A deep knowledge of the imaging needs for each particular TTVI is of paramount importance (Table 3).

\section{Clinical evaluation and heart-team decision}

It is imperative to evaluate the preoperative risk in patients with significant TR amenable for intervention, as is for any cardiac surgery. However, the Society of Thoracic Surgeons (STS) risk score is not suitable for evaluation of patients with TV disease, since it was not originally designed for that purpose. Recently, novel specific risk models to predict morbidity and mortality in patients undergoing TV interventions have been proposed. The LaPar Risk Score, included several clinical (age, sex, stroke, hemodialysis, left ventricular function, chronic lung disease, functional class, reoperation) and operative characteristics, albeit did not consider indicators of right ventricular or liver function (22). More recently, the TRuE Risk Score has been suggested as a prognostic score for stratifying TR patients, based on comorbidities and morphofunctional features of the right cardiac function (https://thetruerisk.com) (23). Furthermore, a specific stepwise assessment considering patient demographics, clinical symptoms, comorbidities, cardiac remodeling and surgical or interventional characteristics has also been proposed (14) (Figure 3).

\section{Transcatheter tricuspid valve interventions}

As discussed before, TR carries a strong impact on survival. However, only few patients are currently referred for TV surgery, which carries a high in-hospital mortality of close to $10 \%(6,7)$. This scenario has led to the development of TTVIs, in many cases adapting devices and lessons learned from percutaneous mitral interventions or mimicking surgical techniques $(16,24,25)$. According to its anatomical target, these therapeutics can be divided in coaptation devices, suture-based or ring-based annuloplasty systems, and orthotopic/heterotopic valve replacement. The ongoing studies of each TTVI are summarized in Table 4.

\section{Coaptation devices}

Coaptation devices aim to minimize the tricuspid leaflet coaptation gap, often encountered in FTR. Three main devices currently allowing transcatheter leaflet repair are the MitraClip (Abbott Vascular, Santa Clara, CA), PASCAL (Edwards Lifesciences, Irvine, CA) and FORMA (Edwards Lifesciences, Irvine, CA) devices (Figure 4A,B).

\section{MitraClip}

Due to the operator familiarity gained over the experience 
Table 1 Grading of severity of chronic TR by echography

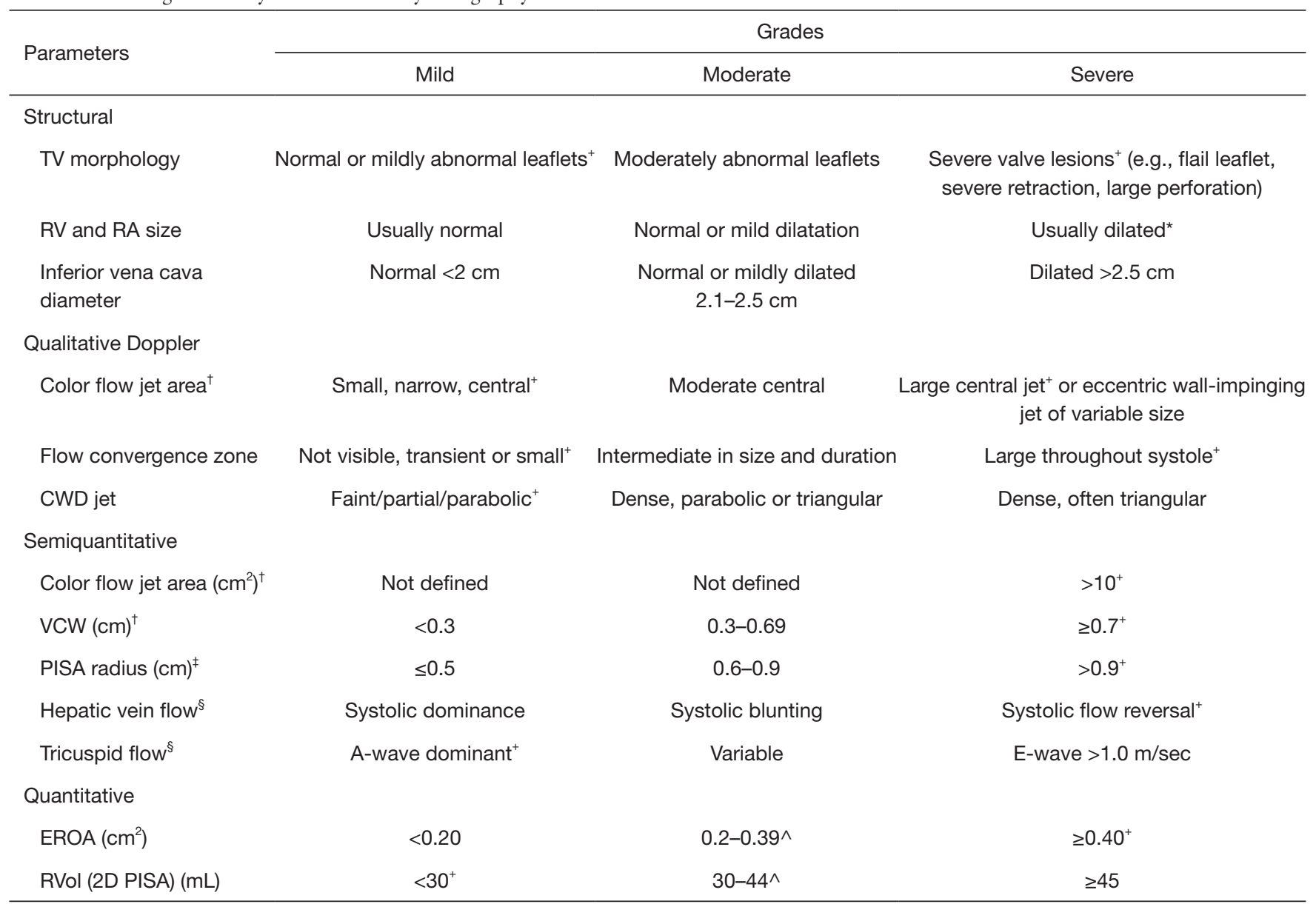

\footnotetext{
${ }^{+}$, signs are considered specific for their TR grades; *, RV anD RA size can be within the "normal" range in patients with acute severe TR;

${ }^{\dagger}$, with Nyquist limit $>50-70 \mathrm{~cm} / \mathrm{sec}$; ${ }^{\ddagger}$, with baseline Nyquist limit shift of $28 \mathrm{~cm} / \mathrm{sec}$; ${ }^{\S}$, signs are nonspecific and are influences by many other factors (RV diastolic function, atrial fibrillation, RA pressure); $\wedge$, there are little data to support further separation of these values. Reproduced with permission from Zoghbi (19). RA, right atrium.
}

in mitral percutaneous interventions, the edge-to-edge repair of the TV has become the most widely TTVI procedure, currently representing close to $70 \%$ of all TTVIs (Figure 4A) (27). In the largest series of tricuspid edge-to-edge repair so far, from the TriValve registry, procedural success (TR reduction to grade $\leq 2+$ ) was achieved in $77 \%$ of patients, with sustained clinical (NYHA functional class $\leq 2$ ) and echocardiographic improvement in $\sim 70 \%$ of the patients at 1 year (28). Of note, concomitant treatment of severe mitral regurgitation was performed in the same procedure in half of the patients. Interestingly, Besler et al. (29) identified the presence of a small TR coaptation gap $(<7.2 \mathrm{~mm})$ and central/anteroseptal TR jet location as independent anatomical predictors of procedural success in patients undergoing TTVI with the MitraClip device.

From a technical point of view, the first clip is preferably aimed to grasp the anterior and septal leaflets. When more than one clip is needed, either bicuspidalization of the tricuspid valve with "zipping" of the anterior and septal leaflets, or the triple orifice technique can be used (30).

The TRILUMINATE study (31) is an ongoing prospective American and European trial specifically addressing the benefits of tricuspid clipping with a slightly modified MitraClip system (TriClip). Enrolment is already complete and the initial 6-month experience has been recently published (32), gathering the information of 85 patients. TR was successfully reduced by at least one grade in $86 \%$ of them at 30 days, while at 6 months of follow-up $4 \%$ of patients suffered a major adverse event 
Table 2 Expansions of the "severe" grade in tricuspid regurgitation

\begin{tabular}{lccccc}
\hline Variable & Mild & Moderate & Severe & Massive & Torrential \\
\hline VC (biplane) & $<3 \mathrm{~mm}$ & $3-6.9 \mathrm{~mm}$ & $7-13 \mathrm{~mm}$ & $14-20 \mathrm{~mm}$ & $\geq 21 \mathrm{~mm}$ \\
EROA (PISA) & $<20 \mathrm{~mm}^{2}$ & $20-39 \mathrm{~mm}^{2}$ & $40-59 \mathrm{~mm}^{2}$ & $60-79 \mathrm{~mm}^{2}$ & $\geq 80 \mathrm{~mm}^{2}$ \\
3D VCA or quantitative EROA & - & - & $75-94 \mathrm{~mm}^{2}$ & $95-114 \mathrm{~mm}^{2}$ & $\geq 115 \mathrm{~mm}^{2}$ \\
\hline
\end{tabular}

a, 3D VCA and quantitative Doppler EROA cut-offs may be larger than PISA EROA. Reproduced with permission from (21). VC, vena contracta; EROA, effective regurgitant orifice área; 3D VCA, three-dimensional vena contracta área.

Table 3 Imaging needs according to the specific transcatheter tricuspid intervention

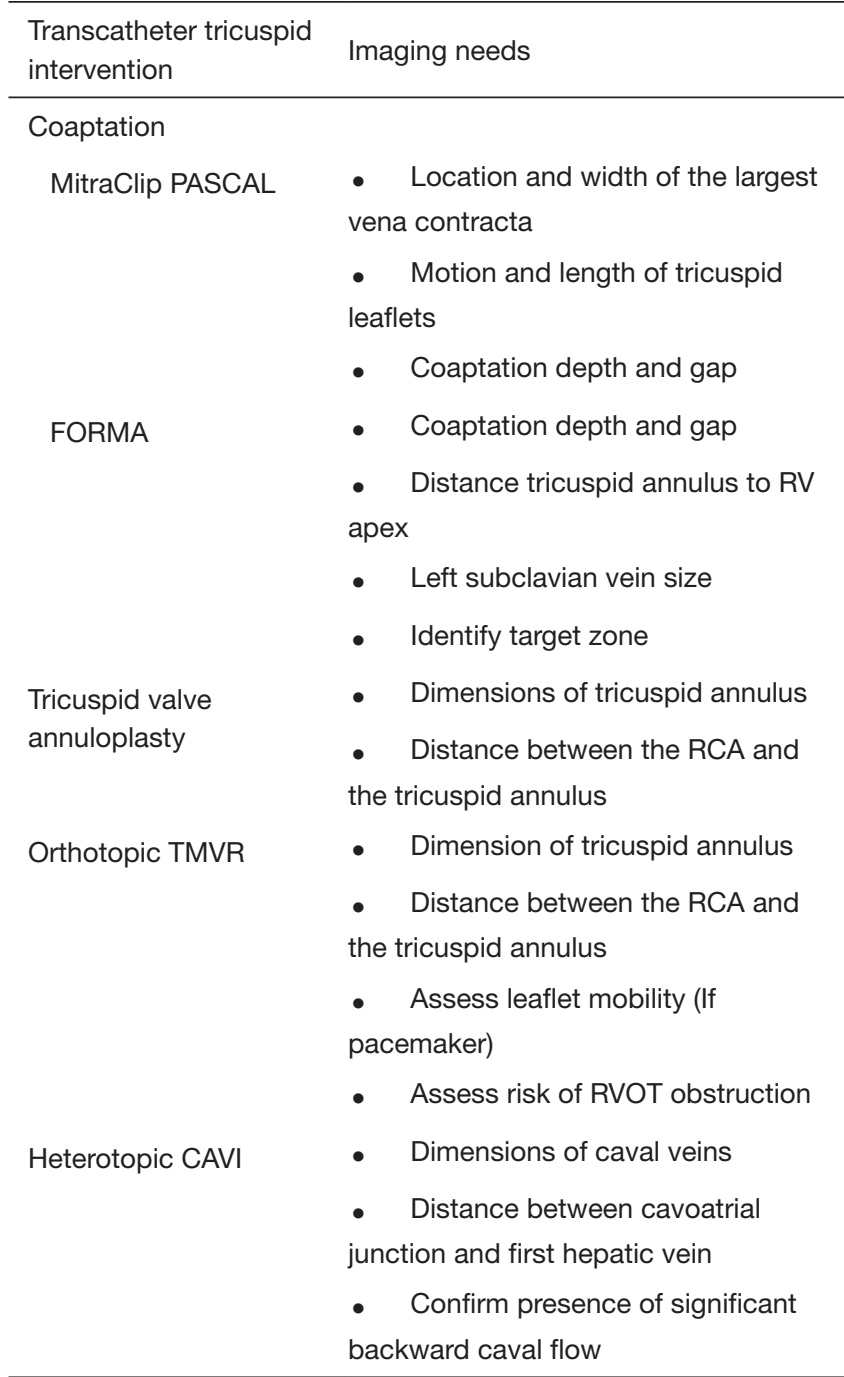

RCA, right coronary artery; RVOT, right ventricular outflow tract. and $5 \%$ died from all causes. Likewise, 1-year outcomes have been recently reported, with sustained improvement in symptoms and quality of life, as well as an improvement in RV function (33). It is worth noting that a different study, the TRILUMINATE Pivotal Trial, is recruiting patients that are being randomized to TriClip versus medical treatment (34).

Most preliminary experiences with edge-to-edge repair thus far have essentially used the MitraClip NTR system. However, the MitraClip XTR, which offers $3 \mathrm{~mm}$ longer clip arms than the NTR system, has emerged as an appealing alternative for treating larger coaptation defects, frequently encountered in FTR patients. A recent experience including 31 patients solely treated with the MitraClip XTR system showed procedural success in $~ 90 \%$ of the patients, with significant improvement at 1 month (35). The device has proven useful to treat FTR without the development of tricuspid stenosis. Nonetheless, up to $25 \%$ of patients with a coaptation gap $\geq 7 \mathrm{~mm}$ experienced a single leaflet clip detachment. Thus, even with larger clips, the previously described discriminator for procedural success of coaptation gap $<7.2 \mathrm{~mm}$ seem to apply (29).

\section{FORMA}

The FORMA repair system consists of a foam-filled balloon allocated in the regurgitant orifice, therefore reducing the leaflet coaptation gap. An anchor in the RV apex confers stability to the system, which is inserted via the subclavian or subaxillary vein (36).

Since the first-in-man experience reported in 2015 (37), long-term outcomes (2-3 years) of 19 patients treated with the FORMA device on a compassionate-use basis have recently been released (38). Notably, procedural success 
Table 4 Ongoing trials of transcatheter tricuspid valve interventions

\begin{tabular}{|c|c|c|c|c|}
\hline Device & Study name & Study design & Patients & Primary endpoints \\
\hline \multirow[t]{2}{*}{ MitraClip } & TRILUMINATE (NCT03227757) & Prospective registry & 85 & Echographic TR reduction $\geq 1$ grade at 30 days \\
\hline & & & & Composite of major events at 6 months \\
\hline PASCAL & CLASP II TR (NCT04097145) & $\begin{array}{l}\text { Prospective } \\
\text { randomized trial }\end{array}$ & 825 & $\begin{array}{l}\text { Composite of major events and improvement of } \\
\text { quality of life at } 24 \text { months }\end{array}$ \\
\hline FORMA & $\begin{array}{l}\text { Early feasibility study of the Edwards } \\
\text { FORMA Tricuspid Transcatheter Repair } \\
\text { System (NCT02471807) }\end{array}$ & Prospective registry & 60 & $\begin{array}{l}\text { Device success and freedom from device- or } \\
\text { procedure-related serious adverse events at } 30 \text { days }\end{array}$ \\
\hline \multirow[t]{2}{*}{ Trialign } & $\begin{array}{l}\text { Early feasibility of the Mitralign PTVAS, } \\
\text { also known as Trialign (NCT02574650) }\end{array}$ & Prospective registry & 30 & All-cause mortality at 30 days \\
\hline & SCOUT II (NCT03225612) & Prospective registry & 60 & All-cause mortality at 30 days \\
\hline TriCinch & $\begin{array}{l}\text { Clinical Trial Evaluation of the } \\
\text { Percutaneous 4Tech TriCinch Coil } \\
\text { Tricuspid Valve Repair System } \\
\text { (NCT03294200) }\end{array}$ & Prospective registry & 90 & $\begin{array}{c}\text { All-cause mortality of the per protocol cohort at } 30 \\
\text { days }\end{array}$ \\
\hline MIA & STTAR (NCT03692598) & Prospective registry & 40 & $\begin{array}{l}\text { Major adverse events and reduction in tricuspid } \\
\text { regurgitation at } 30 \text { days }\end{array}$ \\
\hline DaVingi & $\begin{array}{l}\text { FiH Study of the DaVingi TR System in } \\
\text { the Treatment of Patients with Functional } \\
\text { Tricuspid Regurgitation (NCT03700918) }\end{array}$ & Prospective registry & 15 & $\begin{array}{c}\text { Safety in terms of incidence and severity of device- } \\
\text { related serious adverse effects } \\
\text { Performance of the adjustment device }\end{array}$ \\
\hline $\begin{array}{l}\text { CAVI with } \\
\text { Sapien }\end{array}$ & HOVER (NCT02339974) & Prospective registry & 15 & $\begin{array}{l}\text { Procedural success in terms of device success and } \\
\text { no device/procedure related SAE at } 30 \text { days, and } \\
\text { individual success defined by device success and } \\
\text { clinical outcomes and functional improvements }\end{array}$ \\
\hline $\begin{array}{l}\text { CAVI with } \\
\text { TricValve }\end{array}$ & TRICUS (NCT03723239) & Prospective registry & 10 & $\begin{array}{l}\text { Major adverse events at } 30 \text { days and change in } \\
\text { NYHA functional class at } 6 \text { months }\end{array}$ \\
\hline $\begin{array}{l}\text { Any available } \\
\text { device }\end{array}$ & TriValve (NCT033416166) & Prospective registry & 312 & Cardiovascular death at 30 days \\
\hline
\end{tabular}

CAVI, caval valve implantation; KCCQ, Kansas City Cardiomyopathy Questionnaire; PTVAS, percutaneous tricuspid valve annuloplasty system; TR, tricuspid regurgitation. 


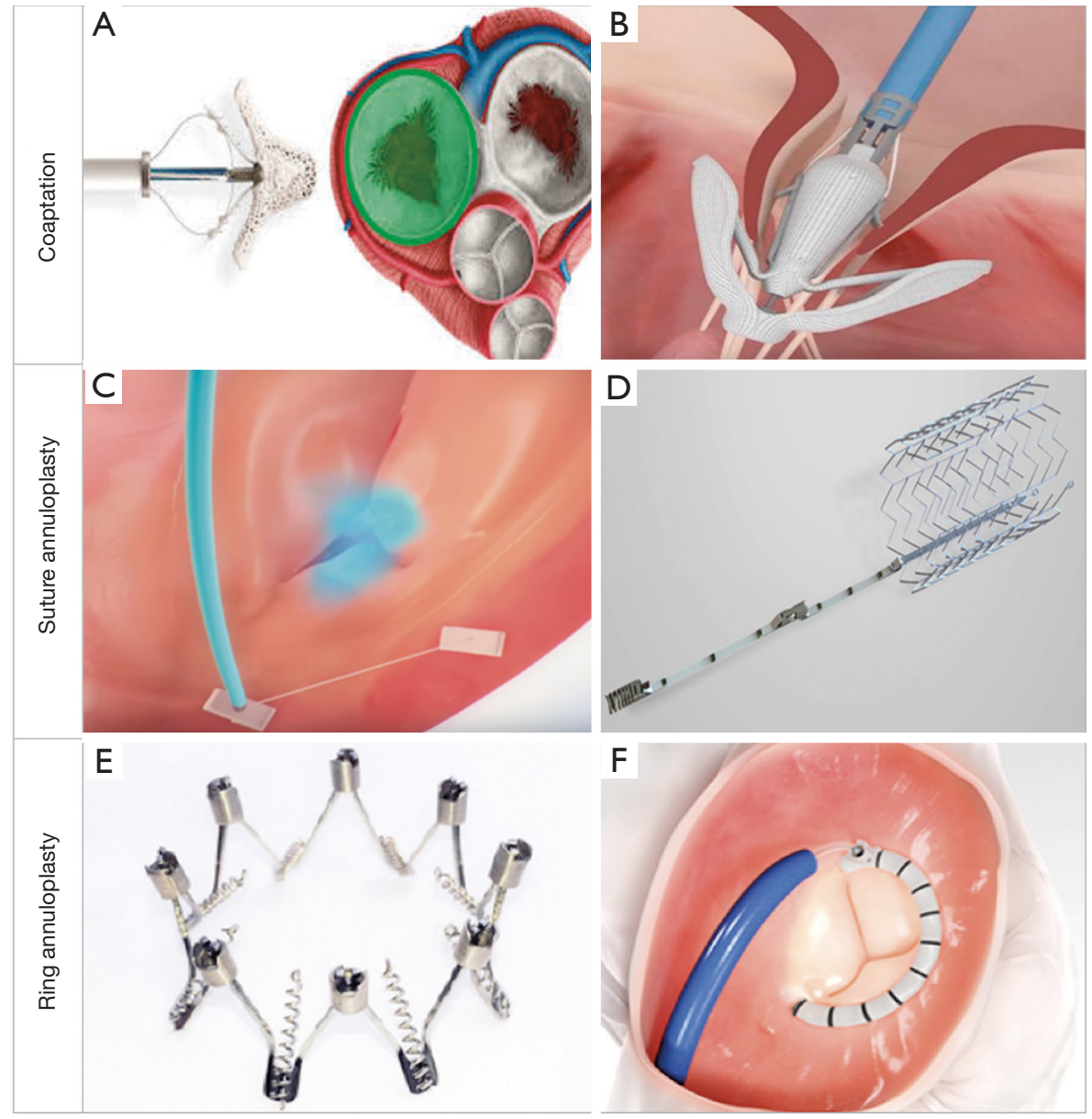

Figure 4 Transcatheter tricuspid repair devices. There are several techniques to percutaneously repair tricuspid regurgitation, which can be classified according to its anatomic target. In the illustration, examples of coaptation devices [MitraClip (A), PASCAL (B)], suture annuloplasty [TriAlign (C), TriCinc (D)] and ring annuloplasty [Milipede IRIS (E), Cardioband (F)]. Reproduced with permission from (26).

was achieved in $89 \%$ of patients, with no deaths at 30 days. Significant improvement of NYHA status and 6MWT was observed at a median follow-up of 32 months. Although severe TR was significantly reduced from $95 \%$ at baseline to $33 \%$ at $2-3$ years, two-thirds of the patients remained with significant TR at last follow-up. Similarly, data from the US early feasibility trial showed significant clinical and echocardiographic improvements at 30 days (39). However, there were 2 procedural deaths due to right ventricular perforation. This led to device enhancements, mainly a new steerable guide sheath to facilitate coaxial alignment to the tricuspid annulus, and an apposition indicator to ensure a more predictable anchoring (40). The US early feasibility trial is ongoing with the device enhancements already adopted (41). The results of the SPACER trial are also awaited to further address the efficacy and safety of the FORMA device (42).

\section{PASCAL}

The PASCAL system combines features from the MitraClip and FORMA devices, since it contains two independently closable grasping paddles (clasps) as well as a central spacer which aims to fill in the regurgitant jet area (Figure 4B) (43). Following the first reported experience (44), a first prospective registry of 28 compassionate use patients has been presented (45). Two patients died in the first 30 days, albeit non-procedural-related. A post-procedural TR grade $\leq 2+$ was achieved in $86 \%$ of the patients which persisted at 30 days. A significant improvement in NYHA functional class was also noted, with $88 \%$ of the patients being in 
NYHA Class $\leq$ II at one-month follow-up, as well as a gain in the 6-minute walking test. As with the MitraClip technique, clasping of the anterior and septal leaflet is the preferred first target with a $66 \%$ ratio.

The first prospective trial designed to evaluate the safety and performance of the PASCAL device is currently enrolling patients (46), whereas launching of a randomized control pivotal trial comparing PASCAL and optical medical therapy (OMT) compared to OMT alone is expected shortly (Table 4) (47).

\section{Annuloplasty}

Transcatheter annuloplasty devices for TR mimic classical surgical techniques aiming to narrow the TA, therefore focusing on the main pathological characteristic that triggers TR worsening (Figure 4C,D,E,F).

\section{Trialign}

The Trialign device (Mitralign, Tewksbury, Massachusetts) recreates the Kay surgical tricuspid bicuspidalization, by means of posterior leaflet obliteration. By transjugular approach, two pledgets are fixed at the posteroseptal and anteroposterior commissures by means of a radiofrequency wire. Next, they are cinched together, therefore plicating the tricuspid posterior leaflet resulting in TV bicuspididalization (Figure 4C) (48). The SCOUT I feasibility trial demonstrated a $100 \%$ implantation success, significant reduction of TA area and EROA, and improvements in functional status at 30 days, in a first cohort of 15 people treated with the Trialign device in the US (20). Twelve-month follow-up showed sustained improvement in symptoms and quality of life (49). The SCOUT II trial (50) will broaden enrollment to USA and Europe with up to 5-year follow-up. A preliminary pooled cohort of 51 SCOUT I and II patients has also been reported, which provide strengthening of the previous data (51). The definitive results of the SCOUT II trial are awaited.

\section{TriCinch}

The TriCinch Coil System (4Tech Cardio, Galway, Ireland) is the second-generation version of the TriCinch device. Its main iteration is that it replaces a corkscrew-shaped anchor for an epicardial coil with two haemostasis seals, to be implanted in the mid-anterior part of the TA. Next, a nitinol stent is placed in the inferior vena cava that is connected to the coil with a Dacron band, therefore generating tension that reduces the TA septolateral diameter and diminishes TR (Figure 4D) (52).

The initial experience is gathered in the PREVENT trial (53), where 24 patients were treated with the firstgeneration device. Successful implantation was achieved in $75 \%$ of them, with $94 \%$ experiencing significant TR reduction and five late anchor detachments. This last issue triggered the aforementioned second-generation device, which has proven safe in the initial in-human experience (54). A prospective registry is currently enrolling patients (55).

\section{PASTA}

The pledget-assisted suture tricuspid annuloplasty (PASTA) emulates the Hetzer's surgical double-orifice repair technique. A "double-bite" is performed and sutures and pledgets are placed at the anterior and septal parts of the TA. The sutures are then tightened using a knot, creating a double-orifice tricuspid valve (56). The first compassionate use in humans has already been reported (57).

\section{MIA}

The minimally invasive annuloplasty (MIA) is a sutureless angioplasty consisting of compliant, self-tensioning PolyCor anchors and the Myolast thermoplastic elastomer. The ongoing STTAR trial is comparing a surgical versus a percutaneous approach for the device implantation (58).

\section{IRIS Millipede}

The IRIS Transcatheter Annuloplasty Ring (Boston Scientific, Marlborough, MA) is a complete, semirigid, zigzag-shaped, adjustable ring made of nitinol (Figure 4E). It is anchored in a supraannular position by means of screws, and then can be adjusted to reduce the diameter of the frame. Two patients have undergone Millipede implantation for tricuspid regurgitation to date, in a combined procedure with mitral regurgitation treatment with the same device. An average $43.5 \%$ reduction of the tricuspid annulus was achieved, as well as complete elimination of TR which lasted at 12 months of follow-up $(59,60)$. A dedicated catheter for TR is still in development.

\section{TRAIPTA}

The transatrial intrapericardial tricuspid annuloplasty (TRAIPTA) system is an indirect tricuspid annuloplasty device where an adjustable circumferential nitinol wire is inserted into the pericardial space via right atrial 

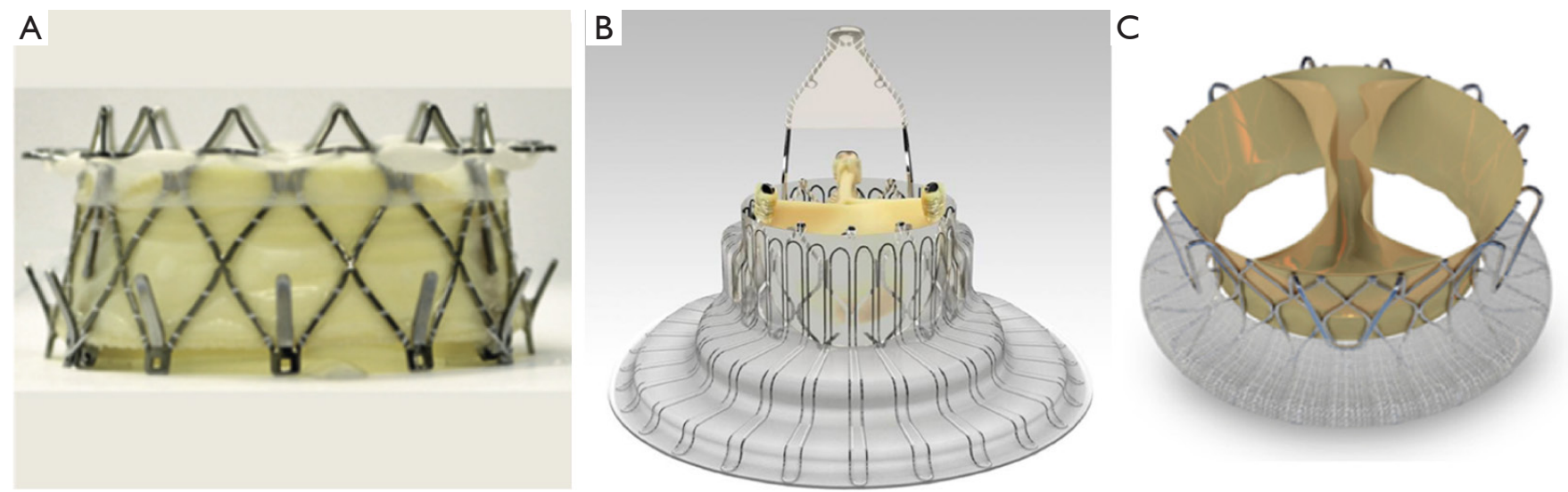

Figure 5 Transcatheter tricusdid replacement devices. Current TTVR prosthesis. (A) Navigate; (B) LUX-Valve; (C) Trisol. Reproduced with permission from (69).

appendage puncture and gets positioned along the right atrioventricular groove. By exerting external pressure, significant longitudinal shortening and leaflet coaptation have been demonstrated in a preclinical pig model (61). After the deployment, the right appendage puncture site is closed with a nitinol atrial septal occluder. The first experience in humans is planned to begin in an upcoming feasibility trial (62).

\section{Cardioband}

The Cardioband System (Edwards Lifesciences, Irvine, CA) is a surgical-like adjustable ring that is implanted on the atrial side of the tricuspid annulus, by advancing multiple repositionable anchors. Next, tailored positioning is achieved by cinching (Figure 4F).

The aim of the TRI-REPAIR Study was to evaluate the performance and safety of the Cardioband in tricuspid position in 30 patients (63). The 1-year results have been reported recently, showing significant reduction in EROA through annular reduction, in addition to improvements in functional status, quality of life and exercise capacity (64).

Concurrently, an Early Feasibility Study is ongoing, for which preliminary 30-day follow-up has also been reported with similar positive outcomes (65). The Cardioband device received CE Mark approval in April 2018 and is currently the only approved device for TTVI in Europe.

\section{DaVingi}

The DaVingi system is a transcatheter device that provides a complete ring annuloplasty for both mitral and tricuspid valves, using a "single-shot" technique of a sole ring implantation with the help of a distal balloon that provides stability. After a period of tissue healing, transcatheter adjustment can be made to ensure proper ring constriction. Preclinical data were reported in 2015 (66) and a firstin-human study in ongoing (67), with six patients already implanted with success. An American Early Feasibility Study in expected for 2020 (68).

\section{Orthotopic TTVR}

\section{GATE}

The GATE system (NaviGate Cardiac Structures, Lake Forest, CA) is a sutureless self-expanding valved-stent dedicated for orthotopic transcatheter tricuspid valve replacement (TTVR). It consists of a nitinol stent, three pericardial leaflets and twelve atrial winglets and ventricular graspers to ensure anchoring (Figure $5 A$ ) (70). Sizes for TA diameters ranging from 36 to $52 \mathrm{~mm}$ are available and is intended to be delivered via transjugular access or transatrial approach trough a minithoracotomy. The initial compassionate-use experience of the first 35 patients treated with the GATE system has been reported recently (71). Procedural success was $77 \%$, with residual $\mathrm{TR} \leq 2+$ in all cases. Surgical conversion was required in $14 \%$ of the patients, and 30-day mortality was $13.8 \%$. Larger studies with longer follow-up are needed to further evaluate the outcomes of the GATE system.

\section{LUX-Valve}

The LUX-Valve (Jenscare Biotechnology, Ningbo, China) is a self-expanding pericardial valve that gets 

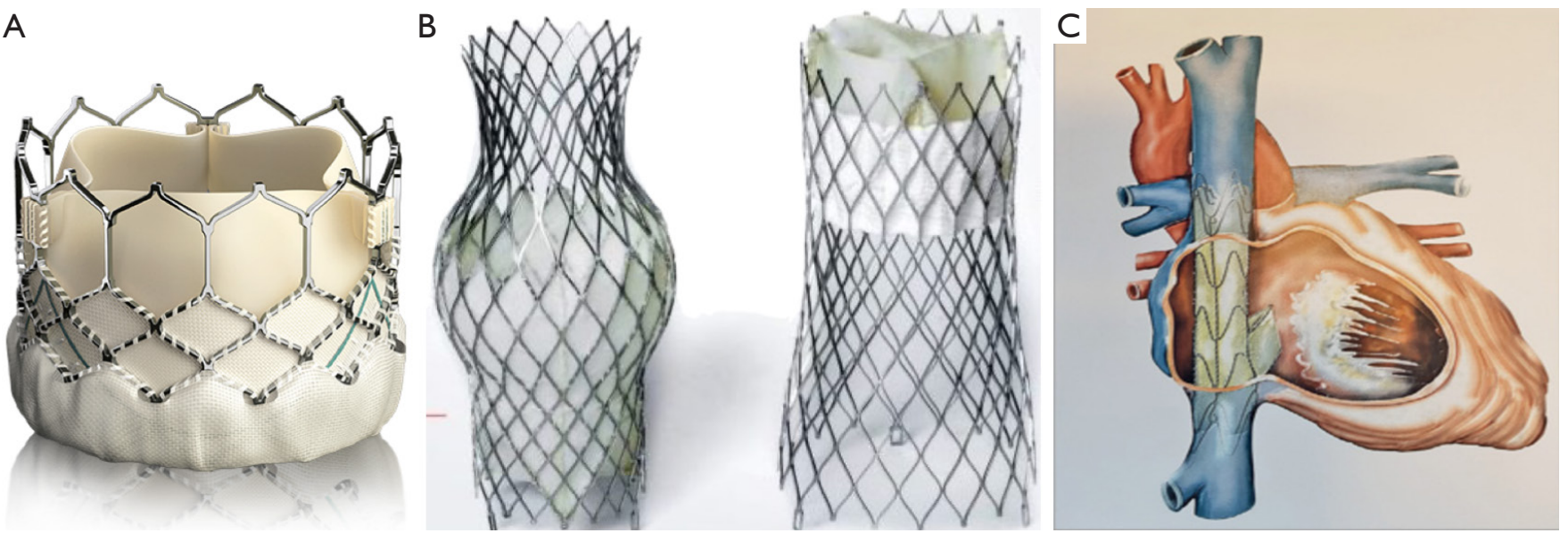

Figure 6 Heterotopic caval valve devices. Current prosthesis for heterotopic caval valve implantation: (A) Edwards Sapien 3; (B) TricValve; (C) Tricento. Reproduced with permission from (74).

enhanced fixation via two anterior leaflet clampers and a specifically designed anchoring mechanism that binds to the interventricular septum (Figure $5 B$ ). It is delivered transatrially via a minimally invasive thoracotomy. The initial compassionate-use experience of 35 patients from three Chinese centers has been reported recently (72). Procedural success was achieved in all patients, with no conversions to open surgery. At 30 days, mortality rate was $5.7 \%$, with significant improvements in NYHA functional class and 6MWT.

\section{TriSol}

The TriSol valve (TriSol Medical, Yokneam, Israel) is mounted on a nitinol self-expanding stent, and it contains a pericardium skirt and a polyester atrial skirt. Its leaflets are dome-shaped, enabling an augmented right ventricle closing volume. At the same time, it contains axial anchors that are designed to avoid conduction disturbances. During diastole, two lateral orifices appear as the two leaflets move to the center of the valve (Figure 5C). This technology is still in a preclinical setting, with positive results arising from 15 acute and 9 chronic implants (up to 5 months of follow-up) in swine models (73). The first human implant is expected soon.

\section{Heterotopic CAVI}

The main focus of heterotopic caval valve implantation (CAVI) is the abolition of the TR-driven venous systemic congestion by implanting balloon-expandable or self-expandable valves in the inferior vena cava or in combination with the superior vena cava (Figure 6). Thus, nor the pathophysiology nor the TR itself are treated with this approach, which is currently reserved for severely symptomatic patients with massive TR non-suitable for other therapeutic options.

\section{SAPIEN}

After an initial successful first-in-man experience in 2013 (75), the performance of an heterotopic 29-mm SAPIEN valve (Edwards Lifesciences, Irvine, CA) (Figure 6A) in the IVC has been tested in a randomized controlled trial versus optimal medical therapy $(76,77)$. Its primary endpoint was exercise capacity measured by maximal oxygen uptake. The enrollment stopped after 28 of the planned 40 patients were enrolled, as the two study arms showed no significant differences in the primary endpoint, and a relevant ratio of complications was registered in the intervention arm. An Early Feasibility Trial of a heterotopic SAPIEN in the IVC is currently underway (78).

\section{TricValve}

The TricValve (P\&F Products \& Features, Vienna, Austria) is a dedicated CAVI valve aiming to solve the issues encountered with off-label heterotopic valves. It consists of a dual self-expandable pericardial tissue valve mounted on nitinol stent frame, one for the IVC and one for the SVC (Figure 6B) (79). Feasibility of the technique was proven for the first time in 2011, succeeding in an immediate abolition of IVC regurgitation and mid-term 
clinical improvement (80). A small study is currently aiming to enroll 10 patients to investigate the safety and performance of the TricValve (81).

\section{Tricento}

The Tricento (NVT, Muri, Switzerland) system consists of a bicavally anchored covered and a lateral pericardium valve that provides inflow to the right atrium (Figure 6C). Since the first-in-human experience in 2018, close to 30 procedures have been successfully performed worldwide $(74,82,83)$. Despite favorable preliminary data with heterotopic CAVI, the long-term clinical impact of leaving TR untreated on right atrial ventricularization, persistent right atrial overload or right ventricular afterload remain unknown and will need to be evaluated in coming trials.

\section{Initial global experience and long-term outcomes}

The patients' characteristics and performance of currently available devices are being evaluated in the TriValve Registry, the largest prospective international registry of various TTVI so far. The last update gathered 312 patients with severe TR undergoing TTVI (27), and is summarized in Table 5. The clinical spectrum depicted a high-risk (mean EuroSCORE II: 9\% $\pm 8 \%$ ), highly symptomatic (NYHA class $\geq$ III: $95 \%$ ) population with prior left-sided valve intervention (35\% of patients) and atrial fibrillation (78\%). FTR was the main etiology in $93 \%$ of the patients, mostly in massive-torrential grades EROA $0.78 \pm 0.60 \mathrm{~cm}^{2}$. Coaptation systems were the most used therapies, particularly MitraClip, accounting for $68 \%$ of the patients; followed by annuloplasty devices (10\% suture annuloplasty systems and 4\% ring annuloplasty systems), heterotopic CAVI (9\%) and GATE and PASCAL systems (3\%). Procedural and 30-day outcomes revealed a $73 \%$ of procedural success and a reasonably low mortality rate of $3.6 \%$. Functional improvement was also noted with $61 \%$ of patients in NYHA class I-II, even with residual TR grade $\geq 3$ in $38 \%$ of the patients. Survival at 1.5 -year follow-up was $77.2 \% \pm 5.9 \%$, being significantly higher among patients in which procedural success was achieved.

The longest and largest follow-up data of patients treated with the MitraClip, FORMA, Trialign, Cardioband and CAVI devices are also outlined in Table 5 $(28,38,49,64,84,85)$. Except for heterotopic CAVI, which included a very high-risk population (median Euroscore of $18.2 \%)$ with high mortality at 1 year (63\%) likely driven by significant comorbidities and the palliative nature of the procedure, patients receiving other TTVI showed similar baseline characteristics (NYHA $\geq$ III 67-96\%; EROA: $\left.0.7-0.92 \mathrm{~cm}^{2}\right)$, high procedural success rates $(X-Y \%)$, with sustained significant clinical improvement $(\mathrm{NYHA} \leq \mathrm{II}$ : 66-90\%) despite modest reductions in TR severity.

\section{Device selection}

A potential strategy for device selection based on the mechanism and natural history of FTR has been proposed (Figure 7) (16). In early phases, where the main mechanism is TA dilatation, an annuloplasty system may be enough to prevent further TA enlargement. Once TA dilatation progresses and leaflet tethering appears, coaptation devices or a combined coaptation and annuloplasty strategy could be used. At this point, TTVR could also prove useful to fully abolish TR. In final stages of the disease, where severe TA dilatation and leaflet tethering ensues, often accompanied by progressive right ventricle dysfunction and remodeling, efforts should be made to detect patients with prohibitive risk and in whom procedures might be potentially futile. In those deemed for intervention, choice between TTVR or CAVI may be considered, probably keeping the latter for patients in end-stage congestive heart failure where only a symptomatic treatment for systemic congestion applies.

\section{Conclusions}

The last decade has seen the advent and widespread adoption of a myriad of TTVI, led by unsatisfactory outcomes of surgical interventions for isolated severe TR. Although initially thought for compassionate use, preliminary data has shown encouraging results, with significant functional and echocardiographic improvements, and may reassure the interventional community to broaden its adoption to a larger spectrum of patients. Indeed, lessons learned from the natural history of this complex valvular disease should entail a rapid recognition of less ill patients in whom a preventive surgical or percutaneous intervention may prove beneficial. Further studies are greatly awaited to provide the necessary background to determine the optimal time and devices to intervene in this less symptomatic population, along with a deeper knowledge of the long-term performance of the variety of technologies currently used in different stages of FTR. 
Table 5 Global experience with transcatheter tricuspid valve interventions

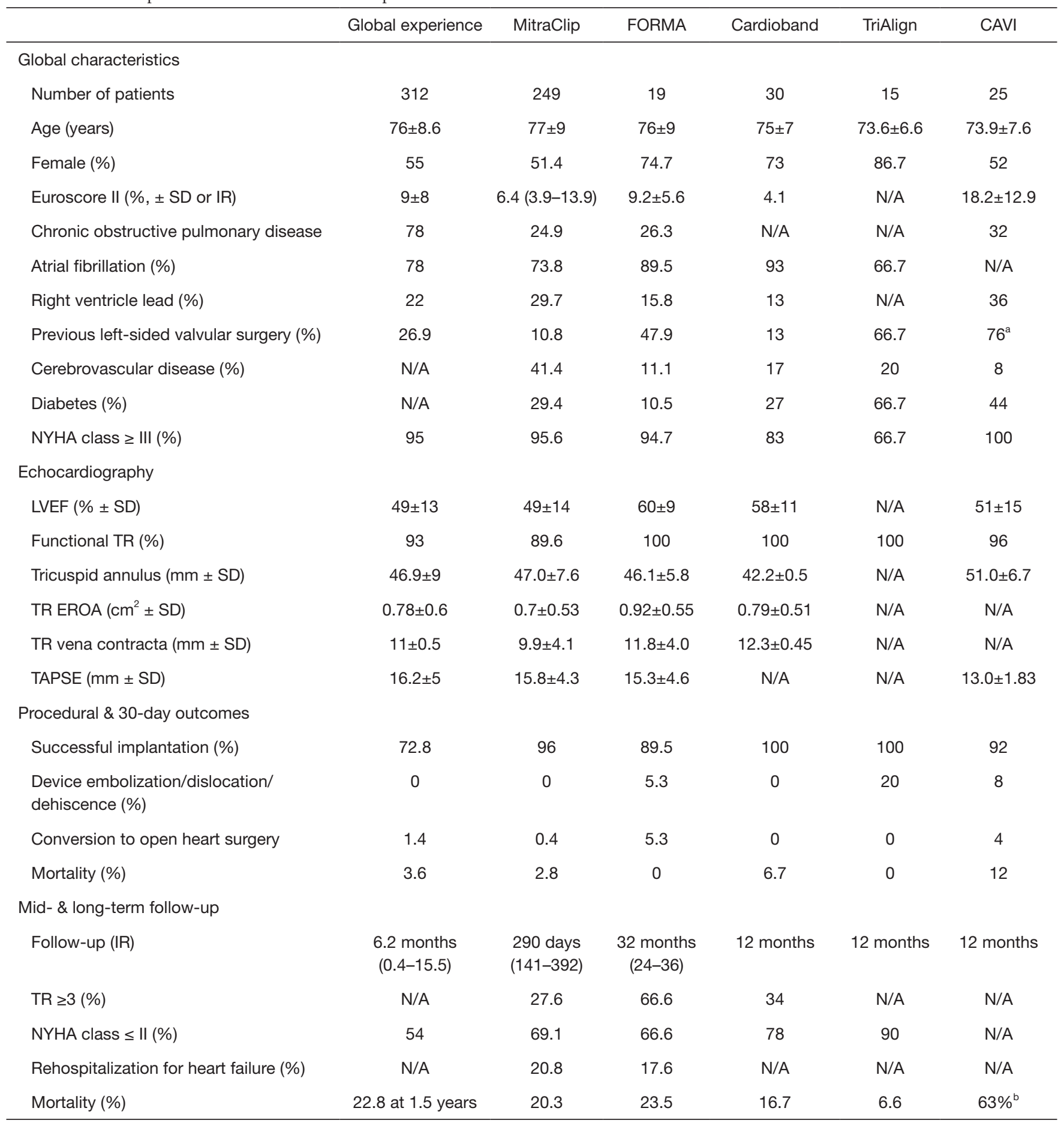

Comparison of the baseline characteristics, initial and long-term outcomes of the TTVIs, alongside global experience derived from the TriValve registry. Data are gathered from references $(27,28,38,49,64,84,85)$. SD, standard deviation; IR, interquartile range; LVEF, left ventricular ejection fraction; TR, tricuspid regurgitation; EROA, effective regurgitation orifice area; TAPSE, tricuspid annulus plane systolic

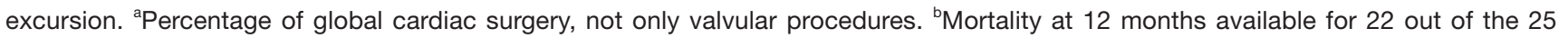
patients in the population. 


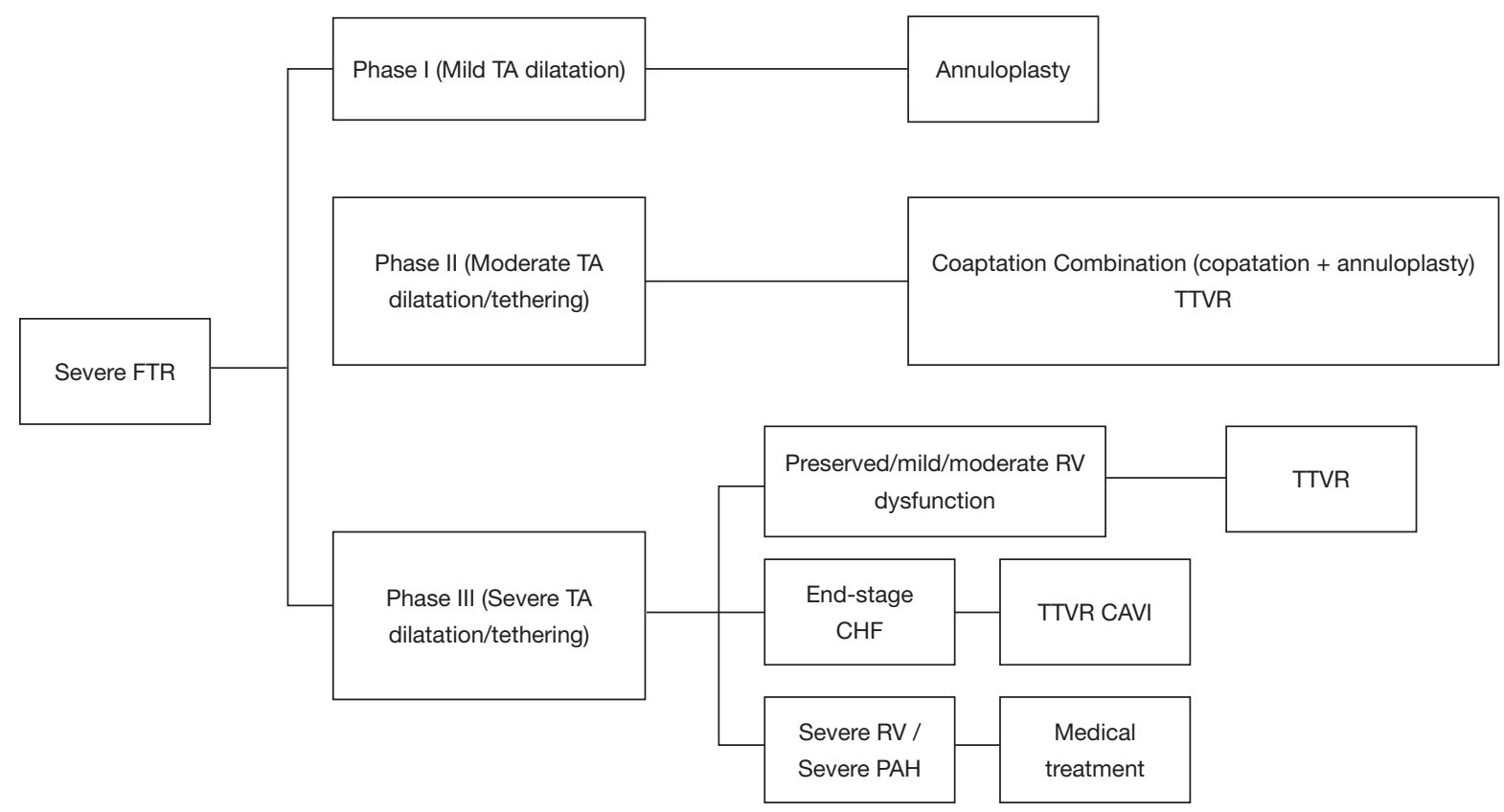

Figure 7 Algorithm for transcatheter tricuspid valve repair in different stages. CAVI, caval valve implantation; FTR, functional tricuspid regurgitation; PH, pulmonary hypertension; TA, tricuspid annulus; TTVR, transcatheter tricuspid valve replacement. Adapted with permission from (16).

\section{Acknowledgments}

Funding: None.

\section{Footnote}

Provenance and Peer Review: This article was commissioned by the Guest Editor (Daniel Hernández-Vaquero) for the series "Structural Heart Disease: The Revolution" published in Annals of Translational Medicine. The article was sent for external peer review organized by the Guest Editor and the editorial office.

Conflicts of Interest: All authors have completed the ICMJE uniform disclosure form (available at http://dx.doi. org/10.21037/atm.2020.03.219). The series "Structural Heart Disease: The Revolution" was commissioned by the editorial office without any funding or sponsorship. DA is proctor for Abbott Medical. The authors have no other conflicts of interest to declare.

Ethical Statement: The authors are accountable for all aspects of the work in ensuring that questions related to the accuracy or integrity of any part of the work are appropriately investigated and resolved.
Open Access Statement: This is an Open Access article distributed in accordance with the Creative Commons Attribution-NonCommercial-NoDerivs 4.0 International License (CC BY-NC-ND 4.0), which permits the noncommercial replication and distribution of the article with the strict proviso that no changes or edits are made and the original work is properly cited (including links to both the formal publication through the relevant DOI and the license). See: https://creativecommons.org/licenses/by-nc-nd/4.0/.

\section{References}

1. Stuge O, Liddicoat J. Emerging opportunities for cardiac surgeons within structural heart disease. J Thorac Cardiovasc Surg 2006;132:1258-61.

2. Nath J, Foster E, Heidenreich PA. Impact of Tricuspid Regurgitation on Long-Term Survival. J Am Coll Cardiol 2004;43:405-9.

3. Wang N, Fulcher J, Abeysuriya N, et al. Tricuspid regurgitation is associated with increased mortality independent of pulmonary pressures and right heart failure: A systematic review and meta-analysis. Eur Heart J 2019;40:476-84.

4. Baumgartner H, Falk V, Bax JJ, et al. 2017 ESC/EACTS 
Guidelines for the management of valvular heart disease. Eur Heart J 2017;38:2739-91.

5. Nishimura RA, Otto CM, Bonow RO, et al. 2014 AHA/ACC Guideline for the Management of Patients With Valvular Heart Disease. J Am Coll Cardiol 2014;63:e57-e185.

6. Zack CJ, Fender EA, Chandrashekar P, et al. National Trends and Outcomes in Isolated Tricuspid Valve Surgery. J Am Coll Cardiol 2017;70:2953-60.

7. Alqahtani F, Berzingi CO, Aljohani S, et al. Contemporary Trends in the Use and Outcomes of Surgical Treatment of Tricuspid Regurgitation. J Am Heart Assoc 2017;6. doi: 10.1161/JAHA.117.007597.

8. Kundi H, Popma JJ, Cohen DJ, et al. Prevalence and Outcomes of Isolated Tricuspid Valve Surgery Among Medicare Beneficiaries. Am J Cardiol 2019;123:132-8.

9. Taramasso M, Pozzoli A, Basso C, et al. Compare and contrast tricuspid and mitral valve anatomy: Interventional perspectives for transcatheter tricuspid valve therapies. EuroIntervention 2018;13:1889-98.

10. van Rosendael PJ, Kamperidis V, Kong WKF, et al. Computed tomography for planning transcatheter tricuspid valve therapy. Eur Heart J 2017;38:665-74.

11. Tornos Mas P, Rodríguez-Palomares JF, Antunes MJ. Secondary tricuspid valve regurgitation: a forgotten entity. Heart 2015;101:1840-8.

12. Rodés-Cabau J, Taramasso M, O'Gara PT. Diagnosis and treatment of tricuspid valve disease: current and future perspectives. Lancet 2016;388:2431-42.

13. Utsunomiya H, Itabashi $\mathrm{Y}$, Mihara $\mathrm{H}$, et al. Functional Tricuspid Regurgitation Caused by Chronic Atrial Fibrillation: A Real-Time 3-Dimensional Transesophageal Echocardiography Study. Circ Cardiovasc Imaging 2017;10. doi: 10.1161/CIRCIMAGING.116.004897.

14. Chang CC, Veen KM, Hahn RT, et al. Uncertainties and challenges in surgical and transcatheter tricuspid valve therapy: a state-of-the-art expert review. Eur Heart J 2020;41:1932-40.

15. Ancona F, Stella S, Taramasso M, et al. Multimodality imaging of the tricuspid valve with implication for percutaneous repair approaches. Heart 2017;103:1073-81.

16. Asmarats L, Puri R, Latib A, et al. Transcatheter Tricuspid Valve Interventions: Landscape, Challenges, and Future Directions. J Am Coll Cardiol 2018;71:2935-56.

17. Prihadi EA, Delgado V, Hahn RT, et al. Imaging Needs in Novel Transcatheter Tricuspid Valve Interventions. JACC Cardiovasc Imaging 2018;11:736-54.

18. Lang RM, Badano LP, Mor-Avi V, et al. Recommendations for cardiac chamber quantification by echocardiography in adults: An update from the American society of echocardiography and the European association of cardiovascular imaging. Eur Heart J Cardiovasc Imaging 2015;16:233-70.

19. Zoghbi WA, Adams D, Bonow RO, et al.

Recommendations for Noninvasive Evaluation of Native Valvular Regurgitation: A Report from the American Society of Echocardiography Developed in Collaboration with the Society for Cardiovascular Magnetic Resonance. J Am Soc Echocardiogr 2017;30:303-71.

20. Hahn RT, Meduri CU, Davidson CJ, et al. Early Feasibility Study of a Transcatheter Tricuspid Valve Annuloplasty: SCOUT Trial 30-Day Results. J Am Coll Cardiol 2017;69:1795-1806.

21. Hahn RT, Zamorano JL. The need for a new tricuspid regurgitation grading scheme. Eur Heart J Cardiovasc Imaging 2017;18:1342-3.

22. LaPar DJ, Likosky DS, Zhang M, et al. Development of a Risk Prediction Model and Clinical Risk Score for Isolated Tricuspid Valve Surgery. Ann Thorac Surg 2018;106:129-36.

23. Lauten A. TRUE Registry: A Prognostic Risk Score for Risk Stratification of Patients With Severe Tricuspid Regurgitation (FTR). In: Presented at TCT 2019.

24. Asmarats L, Taramasso M, Rodés-Cabau J. Tricuspid valve disease: diagnosis, prognosis and management of a rapidly evolving field. Nat Rev Cardiol 2019;16:538-54.

25. Curio J, Demir OM, Pagnesi M, et al. Update on the current landscape of transcatheter options for tricuspid regurgitation treatment. Interv Cardiol 2019;14:54-61.

26. Williams AM, Bolling SF, Latib A. The five Ws of transcatheter tricuspid valve repair: Who, What, When, Where, and Why. EuroIntervention 2019;15:841-5.

27. Taramasso M, Alessandrini H, Latib A, et al. Outcomes After Current Transcatheter Tricuspid Valve Intervention: Mid-Term Results From the International TriValve Registry. JACC Cardiovasc Interv 2019;12:155-65.

28. Mehr M, Taramasso M, Besler C, et al. 1-Year Outcomes After Edge-to-Edge Valve Repair for Symptomatic Tricuspid Regurgitation. JACC Cardiovasc Interv 2019;12:1451-61.

29. Besler C, Orban M, Rommel KP, et al. Predictors of Procedural and Clinical Outcomes in Patients With Symptomatic Tricuspid Regurgitation Undergoing Transcatheter Edge-to-Edge Repair. JACC Cardiovasc Interv 2018;11:1119-28.

30. Braun D, Orban M, Orban M, et al. Transcatheter Edge- 
to-Edge Repair for Severe Tricuspid Regurgitation Using the Triple-Orifice Technique Versus the Bicuspidalization Technique. JACC Cardiovasc Interv 2018;11:1790-2.

31. Anon. US National Library of Medicine. Clinicaltrials.gov 2019. Available online: https://clinicaltrials.gov/ct2/show/ NCT03227757.

32. Nickenig G, Weber M, Lurz P, et al. Transcatheter edgeto-edge repair for reduction of tricuspid regurgitation: 6-month outcomes of the TRILUMINATE single-arm study. Lancet 2019;394:2002-11.

33. Nickening G. Percutaneous Edge-to-Edge Repair for Tricuspid Regurgitation: Initial 1 Year Outcomes from the TRILUMINATE Clinical Trial. In: Presented at PCR London Valves 2019.

34. Anon. US National Library of Medicine. Clinicaltrials.gov 2019. Available online: https://clinicaltrials.gov/ct2/show/ NCT03904147.

35. Braun D, Rommel KP, Orban M, et al. Acute and ShortTerm Results of Transcatheter Edge-to-Edge Repair for Severe Tricuspid Regurgitation Using the MitraClip XTR System. JACC Cardiovasc Interv 2019;12:604-5.

36. Puri R, Rodés-Cabau J. Transcatheter interventions for tricuspid regurgitation: The FORMA Repair System. EuroIntervention 2016;12:Y113-Y115.

37. Campelo-Parada F, Perlman G, Philippon F, et al. First-inMan Experience of a Novel Transcatheter Repair System for Treating Severe Tricuspid Regurgitation. J Am Coll Cardiol 2015;66:2475-83.

38. Asmarats L, Perlman G, Praz F, et al. Long-Term Outcomes of the FORMA Transcatheter Tricuspid Valve Repair System for the Treatment of Severe Tricuspid Regurgitation. JACC Cardiovasc Interv 2019;12:1438-47.

39. Anon. US National Library of Medicine. Clinicaltrials.gov 2019. Available online: https://clinicaltrials.gov/ct2/show/ NCT02471807.

40. Asmarats L, Philippon F, Bédard E, Rodés-Cabau J. FORMA Tricuspid Repair System: device enhancements and initial experience. EuroIntervention 2019;14:1656-7.

41. Hensey M. The FORMA Spacer ... A Versatile Tool Technology and Clinical Updates. In: Presented at TVT Structural Summit June 2019.

42. Anon. US National Library of Medicine. Clinicaltrials.gov 2019. Available online: https://clinicaltrials.gov/ct2/show/ NCT02787408.

43. Praz F, Spargias K, Chrissoheris M, et al. Compassionate use of the PASCAL transcatheter mitral valve repair system for patients with severe mitral regurgitation: a multicentre, prospective, observational, first-in-man study.
Lancet 2017;390:773-80.

44. Fam NP, Ho EC, Zahrani M, et al. Transcatheter Tricuspid Valve Repair With the PASCAL System. JACC Cardiovasc Interv 2018;11:407-8.

45. Kreidel F. Compassionate Use of the PASCAL Transcatheter Valve Repair System for Severe Tricuspid Regurgitation: A Multicenter, Observational, First-inHuman Experience. In: Presented at TVT Structural Summit 14th June 2019.

46. Anon. US National Library of Medicine. Clinicaltrials.gov 2019. Available online: https://clinicaltrials.gov/ct2/show/ NCT03745313.

47. Anon. US National Library of Medicine. Clinicaltrials.gov 2019. Available online: https://clinicaltrials.gov/ct2/show/ NCT04097145.

48. Schofer J, Bijuklic K, Tiburtius C, et al. First-in-human transcatheter tricuspid valve repair in a patient with severely regurgitant tricuspid valve. J Am Coll Cardiol 2015;65:1190-5.

49. Hahn RT. SCOUT I 12-month data. In: Presented at TCT 2017.

50. Anon. US National Library of Medicine. Clinicaltrials.gov 2018. Available online: https://clinicaltrials.gov/ct2/show/ NCT03225612.

51. Meduri CU. SCOUT Study: Trialign results at 30 days from combined US and EU cohort for the treatment of functional TR. In: Presented at TCT September 2018.

52. Latib A, Agricola E, Pozzoli A, et al. First-in-man implantation of a tricuspid annular remodeling device for functional tricuspid regurgitation. JACC Cardiovasc Interv 2015;8:e211-4.

53. Anon. US National Library of Medicine. Clinicaltrials.gov 2018. Available online: https://clinicaltrials.gov/ct2/show/ NCT02098200.

54. Denti P. 4Tech - clinical outcomes and current challenges. In: Presented at PCR London Valves 2017.

55. Anon. Clinical Trial Evaluation of the Percutaneous 4Tech TriCinch Coil Tricuspid Valve Repair System. Clinicaltrials.gov 2019. Available online: https:// clinicaltrials.gov/ct2/show/NCT03294200.

56. Khan JM, Rogers T, Schenke WH, et al. Transcatheter pledget-assisted suture tricuspid annuloplasty (PASTA) to create a double-orifice valve. Catheter Cardiovasc Interv 2018;92:E175-84.

57. Greenbaum AB. Transcatheter tricuspid valve repair: available techniques and patient candidates criteria. In: Presented at CRT 2018.

58. Anon. US National Library of Medicine. Clinicaltrials.gov 
2019. Available online: https://clinicaltrials.gov/ct2/show/ NCT03692598.

59. Rogers J. Transcatheter Tricuspid Valve Therapies 5: Millipede Disclosure Statement of Financial Interest. In: Presented at TCT 2016.

60. Rogers J. Millipede for TR: Technique and Outcomes. In: Presented at TVT 2017.

61. Rogers T, Ratnayaka K, Sonmez M, et al. Transatrial intrapericardial tricuspid annuloplasty. JACC Cardiovasc Interv 2015;8:483-491.

62. Rogers T. TRAIPTA - An update for 2017. In: Presented at TCT 2017.

63. Anon. US National Library of Medicine. Clinicaltrials.gov 2019. Available online: https://clinicaltrials.gov/ct2/show/ NCT02981953.

64. von Bardeleben RS. One-year outcomes of the TRIREPAIR study assessing Cardioband tricuspid valve reconstruction system for patients with functional tricuspid regurgitation. In: Presented at TCT 2019.

65. Anon. US National Library of Medicine. Clinicaltrials.gov 2019. Available online: https://clinicaltrials.gov/ct2/show/ NCT03382457.

66. Kuck KH. Cardiac Implants' Complete Ring Delivery System For Mitral and Tricuspid Valve Annuloplasty. In: Presented at TCT 2015.

67. Anon. FiH Study of the DaVingiTM TR System in the Treatment of Patients With Functional Tricuspid Regurgitation. Clinicaltrials.gov 2018. Available online: https:/clinicaltrials.gov/ct2/show/NCT03700918.

68. Leon MB. Cardiac Implants Da Vingi... Proof of concept. Technology and clinical uptades. In: Presented at TVT 2019.

69. Demir OM, Regazzoli D, Mangieri A, et al. Transcatheter Tricuspid Valve Replacement: Principles and Design. Front Cardiovasc Med 2018;5:129.

70. Navia JL, Kapadia S, Elgharably H, et al. First-in-Human Implantations of the NaviGate Bioprosthesis in a Severely Dilated Tricuspid Annulus and in a Failed Tricuspid Annuloplasty Ring. Circ Cardiovasc Interv 2017;10. doi: 10.1161/CIRCINTERVENTIONS.117.005840.

71. Kapadia S. Navigate Transcatheter Tricuspid Valve Replacement... Early Findings - Technology and Clinical Updates. In: Presented at TVT 2019.

72. Cao P. A New Non-Radial Force Transcatheter Tricuspid Valve Replacement - LuX Valve. In: Presented at TCT 2019.

73. Weisz G. TriSol Percutaneous Transcatheter Tricuspid Valve Replacement. In: Presented at TVT 2019.

74. Toggweiler S, De Boeck B, Brinkert M, et al. First-inman implantation of the Tricento transcatheter heart valve for the treatment of severe tricuspid regurgitation. EuroIntervention 2018;14:758-61.

75. Laule M, Stangl V, Sanad W, Lembcke A, Baumann G, Stangl K. Percutaneous transfemoral management of severe secondary tricuspid regurgitation with edwards sapien XT bioprosthesis: First-in-man experience. J Am Coll Cardiol 2013;61:1929-31.

76. Dreger H. Treatment of Severe TRIcuspid Regurgitation in Patients with Advanced Heart Failure with CAval Vein Implantation of the Edwards Sapien XT VALve (TRICAVAL). In: Presented at TCT 2018.

77. Laule M, Mattig I, Schöbel C, et al. Inferior Caval Valve Implantation Versus Optimal Medical Therapy for Severe Tricuspid Regurgitation. J Am Coll Cardiol 2019;74:4735.

78. Anon. US National Library of Medicine. Clinicaltrials.gov 2019. Available online: https://clinicaltrials.gov/ct2/show/ NCT02339974.

79. Figulla HR, Kiss K, Lauten A. Transcatheter interventions for tricuspid regurgitation - Heterotopic technology: TricValve. EuroIntervention 2016;12:Y116-Y118.

80. Lauten A, Ferrari M, Hekmat K, et al. Heterotopic transcatheter tricuspid valve implantation: first-in-man application of a novel approach to tricuspid regurgitation. Eur Heart J 2011;32:1207-13.

81. Anon. US National Library of Medicine. Clinicaltrials.gov 2019. Available online: https://clinicaltrials.gov/ct2/show/ NCT03723239.

82. Montorfano M, Beneduce A, Ancona MB, et al. Tricento Transcatheter Heart Valve for Severe Tricuspid Regurgitation. JACC Cardiovasc Interv 2019;12:e189-e191.

83. Íñiguez-Romo A, Baz JA, Calvo-Iglesias FE, et al. Percutaneous Tricuspid Valve Replacement Using a Valved Bioprothesis. Rev Esp Cardiol 2019;72:1083-4.

84. Nickenig G, Weber M, Schueler R, et al. 6-Month Outcomes of Tricuspid Valve Reconstruction for Patients With Severe Tricuspid Regurgitation. J Am Coll Cardiol 2019;73:1905-15

85. Lauten A, Figulla HR, Unbehaun A, et al. Interventional Treatment of Severe Tricuspid Regurgitation: Early Clinical Experience in a Multicenter, Observational, Firstin-Man Study. Circ Cardiovasc Interv 2018;11:e006061.

Cite this article as: Santaló-Corcoy M, Asmarats L, Li CH, Arzamendi D. Catheter-based treatment of tricuspid regurgitation: state of the art. Ann Transl Med 2020;8(15):964. doi: 10.21037/atm.2020.03.219 